\title{
Restructuring the McMaster anesthesia clerkship program examination process: a modified approach to multiple choice question examination
}

\author{
Misha Virdee, BSc • Dillon Horth, MBChB - Mary Lou Schmuck, BA • \\ Daniel Cordovani, MD, MSc
}

Received: 13 September 2019/Revised: 7 February 2020/ Accepted: 4 March 2020/Published online: 2 April 2020

(C) Canadian Anesthesiologists' Society 2020

\section{To the Editor,}

All McMaster medical students undertake two weeks of anesthesia training during their clerkship. We believe that the rotation's "traditional" multiple-choice exam tested the students' factual knowledge rather than an understanding of principles. Our goal was to design an exam that better assessed the application of knowledge to a clinical situation.

The traditional examination had flaws typical of many multiple-choice question (MCQ) exams including logical cues (spelling and grammatical errors that allow examinees to eliminate incorrect answers because they do not make grammatical or logical sense), unfocused items (the stem fails to pose a direct question), clang associations (the language used in the question is repeated within the correct response option), convergence strategy (the correct option has the most in common with the other options), and the "except" format (which can interfere with knowledge assessment because of misinterpretation of the question). ${ }^{1}$

We formed a group that included the Anesthesia Undergraduate Program Director, three anesthesia residents, and one medical student. All members completed the "Mac Health Primer on Writing or Editing

M. Virdee, BSc $(\bowtie) \cdot$ D. Horth, MBChB .

M. L. Schmuck, BA · D. Cordovani, MD, MSc

McMaster University, Hamilton, ON, Canada

e-mail: misha.virdee@medportal.ca
Quality MCQ Items" and two completed courses offered by the National Board of Medical Examiners.

Our theoretical framework to revise the MCQ exam was based on principles highlighted in Miller's pyramid of clinical competence, which divides professional authenticity into four categories: knows, knows how, shows, and does. ${ }^{2}$ Our goal was to assess knows how. We eliminated outdated topics and used a Modified Essay Questions format to create a new question bank, in which a question was preceded by a clinical vignette. ${ }^{2}$ Out of the 130 questions in the previous bank, 100 were edited and the remaining 30 were deemed non-salvageable; 72 new questions were created. Of the 100 edited questions, $40 \%$ contained logical cues, $11 \%$ contained unfocused items, $14 \%$ showed clang associations, $10 \%$ showed convergence strategy, and 37\% utilized the except format (Figure).

Criterion-based validity refers to "how well the instrument under consideration compares to certain gold standard measurements." 3 Predictive validity is a subset of criterion validity and can be thought of as the "degree to which a measurement instrument accurately predicts theoretically expected outcomes". We believe that the results from the modified exam have increased construct validity with regard to their reflection of the student's assimilation and understanding of the information provided during their anesthesia clerkship rotation. We share our experience with the hope of helping academic centres to achieve the goal of assessing the category knows how.

Our new exam addresses the design flaws common to many MCQs. We hope that these efforts can serve as a stimulus for other undergraduate anesthesia programs to reconsider their MCQ examinations. Future plans include a comparison of the new exam scores with old exam scores and assessment of how they correlate with clinical performance of anesthesia trainees. 
(a)

Old Question Pre Editing:

The following are risk factors for aspiration at induction of general anesthesia EXCEPT:
a. Obesity
b. Hypertension
c. Pregnancy
d. Bowel Obstruction
e. Insulin Dependant Diabetes Mellitus

\section{Question Post Editing:}

You are about to induce a 35 year old female for a routine general surgery operation. You are reviewing her risk factors for aspiration. Patients with which of the following conditions would be most appropriate for a titrated induction as opposed to a rapid sequence induction?
a. Recent meal
b. Hypertension
c. Pregnancy
d. Bowel obstruction

(b)

Completely New Question:

You are providing a general anesthetic for a 48 year old woman having a vaginal hysterectomy. Shortly after induction, her vital signs are as shown below. Which pharmacological intervention is most appropriate?
a. Administer phenylephrine
b. Administer esmolol
c. Administer ephedrine
d. Administer vasopressin

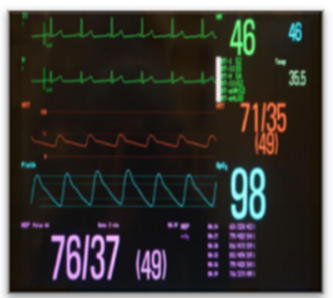

(c) Which of the following statements is true?
a. A patient with ASA 2 classification has mild systemic disease and can be operated on as an outpatient
b. Restricting food for 8 hours before surgery always eliminates risk of aspiration
c. Risk of a post-dural puncture decreases with increasing size of the spinal needle
d. None of the above

(d) The following is / are true:
a. Infants and children have rate dependent cardiac output
b. Hypoxemia is an important cause of bradycardia in children
c. Infants have a greater blood volume $/ \mathrm{kg}$ than children
d. All of the above

(e) Which of the following statements regarding the ASA Physical Classification System is true?
a. Class $\mathrm{V}$ denotes a patient in excellent health
b. It is used for patients taking aspirin
c. The addition of an E suffix to the class denotes an elective surgical case
d. It is a classification system designed to standardise the overall state of health of the surgical patient

(f) You have just placed an endotracheal tube. The patient's abdomen is distending, there are no breath sounds and gurgling sound is heard with ventilation. $\mathrm{SpO} 2$ is $100 \%$. What is your next action?
a. Remove the tube and attempt a blind intubation
b. Remove the tube and ventilate the patient
c. Remove the tube halfway and try to ventilate the patient
d. Leave the tube in blocking the esophagus and attempt to place a new endotracheal tube in the trachea
e. Ventilate the patient and proceed to attempt an emergency front of neck access 
4 Figure A) Example of a question edited where the "except" flaw was eliminated and a clinical stem was added. B) Example of a question created to assess knowledge application (in clinical practice). C) Example of a question with a "logical cue" flaw. D) Example of a question with an "unfocused items" flaw. E) Example of a question with a "clang association" flaw. F) Example of a question with a "convergence strategy" flaw

\section{Conflicts of interest None.}

Funding statement None.

Editorial responsibility This submission was handled by Dr. Steven Backman, Associate Editor, Canadian Journal of Anesthesia.

\section{References}

1. Medical Council of Canada. Guidelines for the Development of Multiple-Choice Questions; 2010. Available from URL: https:// mcc.ca/media/Multiple-choice-question-guidelines.pdf (accessed March 2020).

2. Miller GE. The assessment of clinical skills/competence/ performance. Acad Med 1990; 65(9 Suppl): S63-7.

3. Thomas PA, Kern DE, Hughes MT, Chen BY. Curriculum Development for Medical Education - a Six Step Approach Third Edition. Johns Hopkins University Press; 2016.

Publisher's Note Springer Nature remains neutral with regard to jurisdictional claims in published maps and institutional affiliations. 